\title{
Mixed Finite Volume Element Method for Vibration Equations of Beam with Structural Damping
}

\author{
Tongxin Wang, Ziwen Jiang, Zhe Yin* \\ College of Mathematics and Statistics, Shandong Normal University, Jinan, China \\ Email: ^ZYin_sdnu@163.com
}

How to cite this paper: Wang, T.X., Jiang, Z.W. and Yin, Z. (2021) Mixed Finite Volume Element Method for Vibration Equations of Beam with Structural Damping. American Journal of Computational Mathematics, 11, 207-225.

https://doi.org/10.4236/ajcm.2021.113014

Received: September 2, 2021

Accepted: September 25, 2021

Published: September 28, 2021

Copyright $\odot 2021$ by author(s) and Scientific Research Publishing Inc. This work is licensed under the Creative Commons Attribution International License (CC BY 4.0).

http://creativecommons.org/licenses/by/4.0/

\begin{abstract}
In this paper, for the initial and boundary value problem of beams with structural damping, by introducing intermediate variables, the original fourthorder problem is transformed into second-order partial differential equations, and the mixed finite volume element scheme is constructed, and the existence, uniqueness and convergence of the scheme are analyzed. Numerical examples are provided to confirm the theoretical results. In the end, we test the value of $\delta$ to observe its influence on the model.
\end{abstract}

\section{Keywords}

Vibration Equations, Structural Damping, Mixed Finite Volume Element Method, Error Estimation, Numerical Simulation

\section{Introduction}

The beam is the most important part of the upper frame of a building, which is widely used in engineering projects, bridge construction and aerospace. So the vibration and damping of beam have always been the concern of engineers and researchers [1] [2] [3]. As we know, the differential equation that the deflection curve of the beam should meet is:

$$
\frac{\mathrm{d}^{2}}{\mathrm{~d} x^{2}}\left(E I \frac{\mathrm{d}^{2} u}{\mathrm{~d} x^{2}}\right)=p(x)
$$

where, $u(x)$ is a displacement of the beam, $p(x)$ is a load force normal to the beam at the point $x, E$ is Young's modulus, and $I$ is the area moment of inertia of the beam's cross-section. There are many research results on beam vibration, both in mechanics and mathematics. Thankane studied the vibration equa- 
tion of a beam with free ends by using the finite differential method in literature [4]. In literature [5] [6] [7], Gupta studied the existence and uniqueness of solutions for a class of beam vibration equations. Ni et al. [8] applied the generalized difference method to solve the free vibration problem of beams by using the first-order element hat function as the trial function and the pieced linear function as the test function, and finally proposed a sixth-order implicit scheme. $\mathrm{Xu}$ et al. [9] constructed a finite difference scheme for the fourth-order equation of the beam, and realized the stable explicit calculation of the approximate solution by using the asymmetric scheme. Zeng put forward the fourth-order difference scheme with two parameters $\alpha$ and $\beta$ [10], the four-layer weighted $\beta$ scheme and the tridiagonal four-layer implicit scheme [11] for the linear equation of the beam, and studied the stability of the difference scheme with the Fourier analysis method. Yin et al. [12] proposed a finite element method to study the vibration of beams on elastic foundations under moving loads using the Hermite element. Catal [13] used the differential transformation method to solve the differential equation of free vibration of a beam on an elastic foundation with fixed end and simply supported end, and obtained the analytical solution and frequency factor. In literature [14], a high-precision multi-parameter numerical scheme for solving the vibration equation of a beam is presented. Sabitov studied the Cauchy problem of beam vibration equations and obtained sufficient conditions for the existence of an explicit solution in the literature [15]. The study of damped elastic systems was probably started by Chen and Russell [16] in 1981. They present a mathematical model exhibiting the empirically observed damping rates in elastic systems. The form of the model studied is as follows:

$$
u_{t t}+B u_{t}+A u=0
$$

where $A$ is the elastic operator and $B$ is the damping operator. In recent years, Fan studied the existence, uniqueness and regularity of the abstract model for the vibration equations of the beam with structural damping and the existence of the global mild solution in literature [17] [18]. Tang and Yin studied the Hermite finite element method for a class of viscoelastic beam vibration problems in the literature [19]. The structural damped vibration of a uniform simply supported beam with length $\mathrm{L}$ can be written in the following one-dimensional fourthorder initial and boundary value problems:

$$
\begin{cases}\text { (a) } u_{t t}-\delta u_{x x t}+u_{x x x x}=f(x, t), & (x, t) \in \Omega \times(0, T] \\ \text { (b) } u(x, 0)=\varphi(x), u_{t}(x, 0)=\psi(x), & x \in \Omega \\ \text { (c) } u(0, t)=u(L, t)=0, u_{x}(0, t)=u_{x}(L, t)=0, & t \in[0, T]\end{cases}
$$

where $u(x, t)$ is used to represent displacement, $\Omega=(0, L), 0<T<\infty, \delta(>0)$ is structural damping coefficient, the source term $f(x, t)$, initial value functions $\varphi(x)$ and $\psi(x)$ are the known smooth functions, $\varphi(x)$ and $\psi(x)$ represent the displacement and velocity of the beam at the initial moment, respectively. 
The objective of this paper is to construct a mixed finite volume element scheme for the vibration problem of the beam with structural damping (3). This scheme was first proposed by Russell [20] in 1995 when he solved the second-order elliptic problem. Then, Jones [21] [22] verified the method with numerical examples. This scheme has the following advantages: firstly, the smoothness of the solution space of the proposed mixed finite volume element method is lower than that of the common finite element method, so it is easier to construct the mixed finite element space; secondly, the mixed finite volume element method can be used to obtain two unknowns at the same time to reduce the cost of calculation; thirdly, the scheme maintains the local conservation of physical quantities. Therefore, based on the above advantages, mixed finite volume element method has been widely used in practical problems. Wang [23] studied the equilibrium equation of beams by using the mixed finite volume element method and proved that the scheme has first-order accuracy in the discrete $H_{1}$ half-norm and the discrete $L^{2}$ norm. In recent years, Yuan et al. [24] [25] used the mixed finite volume element method to simulate the transient behavior of semiconductor heat transfer devices and to solve the oil-water two-phase displacement problem.

The paper is organized as follows: in Section 2, the vibration equation of structural damping beams (3) is transformed into second-order equations with similar ideas in [8]. Then, the spatial derivative term is discretized by the mixed finite volume element method, and the time derivative term is discretized by the backward Euler scheme to construct the mixed finite volume element scheme of (3); in Section 3, some necessary lemmas are given; in Section 4, we prove the existence and uniqueness of solutions of mixed finite volume element scheme; The convergence of semi-discrete and fully-discrete mixed finite volume element schemes is proved in Sections 5 and 6 respectively; in Section 7, some numerical examples are given to verify the accuracy of the scheme, which indicates that the scheme has high practicability.

\section{Mixed Finite Volume Element Scheme}

In order to formulate the mixed finite volume element approximate scheme, we introduce two auxiliary variables:

$$
v(x, t)=u_{t}(x, t), \quad w(x, t)=u_{x x}(x, t)
$$

$v(x, t)$ and $w(x, t)$ denotes the speed and bending moment of the beam when it vibrates respectively, then (1) can be rewritten as:

$$
\begin{cases}\text { (a) } v-\delta v_{x x}+w_{x x}=f(x, t), & (x, t) \in \Omega \times(0, T] \\ \text { (b) } v_{x x}=w_{t}, & (x, t) \in \Omega \times(0, T] \\ \text { (c) } v(x, 0)=\psi(x), w(x, 0)=\varphi^{\prime \prime}(x), & x \in \Omega \\ \text { (d) } v(0, t)=v(L, t)=0, & t \in[0, T]\end{cases}
$$

Multiply the Equations (4)(a) and (4)(b) by $\phi \in H_{0}^{1}(\Omega)$ respectively and in- 
tegrate them on $\Omega$. Applying Green's formula [26], we can get a weak form equivalent to (3): Find $(v, w):[0, T] \rightarrow H_{0}^{1}(\Omega) \times H^{1}(\Omega)$, such that:

$$
\left\{\begin{array}{l}
\text { (a) }\left(v_{t}, \phi\right)+\delta\left(v_{x}, \phi_{x}\right)-\left(w_{x}, \phi_{x}\right)=(f, \phi), \quad \forall \phi \in H_{0}^{1}(\Omega) \\
\text { (b) }\left(w_{t}, \phi\right)+\left(v_{x}, \phi_{x}\right)=0, \quad \forall \phi \in H^{1}(\Omega) \\
\text { (c) } v(x, 0)=\psi(x), \quad x \in \Omega \\
\text { (d) } w(x, 0)=\varphi^{\prime \prime}(x), \quad x \in \Omega
\end{array}\right.
$$

Next, we introduce the semi-discrete mixed finite volume element scheme of (1).

Let $0=x_{0}<x_{1}<x_{2}<\cdots<x_{N}=L$ be the primal partition of $\bar{\Omega}$, the corresponding dual partition is $0=x_{0}<x_{\frac{1}{2}}<x_{\frac{3}{2}}<\cdots<x_{N-\frac{1}{2}}<x_{N}=L$, where:

$x_{i+\frac{1}{2}}=\frac{x_{i}+x_{i+1}}{2},(i=0,1,2, \cdots, N-1)$.

Now we choose a quasi-uniform subdivision $\mathfrak{J}_{h}=\left\{A_{i}=\left[x_{i}, x_{i+1}\right] ; i=0,1,2 \cdots N-1\right\}$ of the region $\Omega$, The diameter of unit $A_{i}$ is $h_{i}=x_{i+1}-x_{i}$, let $h=\lim _{0 \leq i \leq N-1} h_{i}$.

We define the dual subdivision as follows:

$$
\mathfrak{I}_{h}^{*}=\left\{A_{i}^{*}=\left[x_{i-\frac{1}{2}}, x_{i+\frac{1}{2}}\right] ; i=0,1,2, \cdots, N-1\right\}
$$

where $A_{0}^{*}=\left[x_{0}, x_{\frac{1}{2}}\right], A_{N}^{*}=\left[x_{N-\frac{1}{2}}, x_{N}\right], A_{i}^{*}$ constitutes the dual unit or control volume of node $i$, For the boundary node, its control volume is modified accordingly.

Define the finite element spaces by:

$$
\begin{gathered}
U_{h}=\left\{u_{h} \in C(\bar{\Omega}),\left.u_{h}\right|_{A} \in P_{1}, \forall A \in \mathfrak{I}_{h}\right\} \\
V_{h}=\left\{v_{h} \in L^{2}(\Omega),\left.v_{h}\right|_{A^{*}} \in P_{0}, \forall A^{*} \in \mathfrak{J}_{h}^{*}\right\} \\
U_{0 h}=\left\{u_{h} \in U_{h}, u_{h}(0)=u_{h}(L)=0\right\} \\
V_{0 h}=\left\{v_{h} \in V_{h}, v_{h}(0)=v_{h}(L)=0\right\}
\end{gathered}
$$

where $U_{h}$ represent the linear finite element space corresponding to the primal partition $\mathfrak{J}_{h}, V_{h}$ is the constant function space of corresponding dual partition $\mathfrak{I}_{h}^{*}$.

The interpolation projection operator $\Pi_{h}^{*}: U_{h} \rightarrow V_{h}$ is defind as:

$$
\Pi_{h}^{*} w_{h}=\sum_{i=1}^{N-1} w_{h}\left(x_{i}\right) \chi_{A_{i}^{*}}, \forall w_{h} \in U_{h}
$$

$\chi_{i}$ represents the eigenfunction on $A_{i}^{*}$, i.e.:

$$
\chi_{A_{i}^{*}}= \begin{cases}1 & x \in A_{i}^{*} \\ 0 & x \notin A_{i}^{*}\end{cases}
$$


Integrate (4) on the dual unit $A_{i}^{*}$ to obtain:

$$
\left\{\begin{array}{l}
\text { (a) } \int_{A^{*}} v(x, t) \mathrm{d} x-\delta\left[v_{x}\left(x_{i+\frac{1}{2}}, t\right)-v_{x}\left(x_{i-\frac{1}{2}}, t\right)\right]+\left[w_{x}\left(x_{i+\frac{1}{2}}, t\right)-w_{x}\left(x_{i-\frac{1}{2}}, t\right)\right] \\
\quad=\int_{A^{*}} f(x, t) \mathrm{d} x \\
\text { (b) } \int_{A^{*}} w_{t}(x, t) \mathrm{d} x-\left[v_{x}\left(x_{i+\frac{1}{2}}, t\right)-v_{x}\left(x_{i-\frac{1}{2}}, t\right)\right]=0
\end{array}\right.
$$

Sum over all the elements and notice that:

$$
\left(w_{h}, \Pi_{h}^{*} \phi_{h}\right)=\phi_{h} \sum_{i=1}^{N-1} \int_{A_{i}^{*}} w_{h} \mathrm{~d} x, \quad \forall w_{h}, \phi_{h} \in U_{0 h}
$$

Define:

$$
B\left(w_{h}, \Pi_{h}^{*} \phi_{h}\right)=-\phi_{h} \sum_{i=1}^{N-1}\left[w_{h x}\left(x_{i+\frac{1}{2}}, t\right)-w_{h x}\left(x_{i-\frac{1}{2}}, t\right)\right], \quad \forall w_{h}, \phi_{h} \in U_{0 h}
$$

Then we have:

$$
\left\{\begin{array}{l}
\text { (a) }\left(v_{t}, \Pi_{h}^{*} \phi_{h}\right)+\delta B\left(v, \Pi_{h}^{*} \phi_{h}\right)-B\left(w, \Pi_{h}^{*} \phi_{h}\right)=\left(f, \Pi_{h}^{*} \phi_{h}\right), \quad \forall \phi_{h} \in U_{0 h} \\
\text { (b) }\left(w_{t}, \Pi_{h}^{*} \phi_{h}\right)+B\left(v, \Pi_{h}^{*} \phi_{h}\right)=0, \quad \forall \phi_{h} \in U_{h}
\end{array}\right.
$$

Then, the semi-discrete mixed finite volume element scheme of problem (3) is: Find $\left(v_{h}, w_{h}\right) \in U_{0 h} \times U_{h}$, such that:

$$
\left\{\begin{array}{l}
\text { (a) }\left(v_{h t}, \Pi_{h}^{*} \phi_{h}\right)+\delta B\left(v_{h}, \Pi_{h}^{*} \phi_{h}\right)-B\left(w_{h}, \Pi_{h}^{*} \phi_{h}\right)=\left(f, \Pi_{h}^{*} \phi_{h}\right), \quad \forall \phi_{h} \in U_{0 h} \\
\text { (b) }\left(w_{h t}, \Pi_{h}^{*} \phi_{h}\right)+B\left(v_{h}, \Pi_{h}^{*} \phi_{h}\right)=0, \quad \forall \phi_{h} \in U_{h} \\
\text { (c) } v_{h}(x, 0)=\psi_{h}(x), \quad x \in \Omega \\
\text { (d) } w_{h}(x, 0)=\varphi_{h}^{\prime \prime}(x), \quad x \in \Omega
\end{array}\right.
$$

\section{Some Lemmas}

In this part, we will give some necessary lemmas. Let $u_{h} \in U_{0 h}$, we define the following norms:

$$
\begin{gathered}
\left\|u_{h}\right\|_{0, h}^{2}=\sum_{i=1}^{N-1} h u_{i}^{2} \\
\left\|u_{h}\right\|_{1, h}^{2}=\left\|u_{h}\right\|_{0, h}^{2}+\left|u_{h}\right|_{1, h}^{2} \\
\left|u_{h}\right|_{1, h}^{2}=\sum_{i=1}^{N} h\left(\frac{u_{i}-u_{i-1}}{h}\right)^{2}
\end{gathered}
$$

Lemma 3.1. [26] On $U_{h}$, the following pairs of norms are equivalent respectively: $|\cdot|_{1, h}$ and $|\cdot|_{1} ;\|\cdot\|_{0, h}$ and $\|\cdot\| ;\|\cdot\|_{1, h}$ and $\|\cdot\|_{1}$. That is, there are positive constants $C_{1}, \cdots, C_{4}$ which is independent of $U_{h}$, such that:

$$
\begin{aligned}
& C_{1}\left\|\psi_{h}\right\|_{0, h} \leq\left\|\psi_{h}\right\| \leq C_{2}\left\|\psi_{h}\right\|_{0, h}, \quad \forall \psi_{h} \in U_{0 h} \\
& C_{3}\left\|\psi_{h}\right\|_{1, h} \leq\left\|\psi_{h}\right\|_{1} \leq C_{4}\left\|\psi_{h}\right\|_{1, h}, \quad \forall \psi_{h} \in U_{0 h}
\end{aligned}
$$


Lemma 3.2. [27] The bilinear form $B\left(\cdot, \Pi_{h}^{*} \cdot\right)$ meets:

$$
\begin{gathered}
B\left(\psi_{h}, \Pi_{h}^{*} \varphi_{h}\right)=B\left(\varphi_{h}, \Pi_{h}^{*} \psi_{h}\right), \quad \forall \psi_{h}, \varphi_{h} \in U_{h} \\
B\left(\psi_{h}, \Pi_{h}^{*} \psi_{h}\right) \geq \frac{1}{2}\left|\psi_{h}\right|_{1}^{2}, \quad \forall \psi_{h} \in U_{0 h} \\
B\left(\psi_{h}, \Pi_{h}^{*} \varphi_{h}\right) \leq C\left|\psi_{h}\right|_{1} \cdot\left|\varphi_{h}\right|_{1}, \quad \forall \psi_{h}, \varphi_{h} \in U_{0 h}
\end{gathered}
$$

where $C>0$ is a constant independent of $h$.

Lemma 3.3. [27] $\left(\cdot, \Pi_{h}^{*} \cdot\right)$ meets:

$$
\begin{gathered}
\left(\psi_{h}, \Pi_{h}^{*} \varphi_{h}\right)=\left(\varphi_{h}, \Pi_{h}^{*} \psi_{h}\right), \quad \forall \psi_{h}, \varphi_{h} \in U_{h} \\
\left(\psi_{h}, \Pi_{h}^{*} \psi_{h}\right) \geq \frac{1}{8}\left\|\psi_{h}\right\|^{2}, \quad \forall \psi_{h} \in U_{h} \\
\left(w, \Pi_{h}^{*} \psi_{h}\right) \leq\|w\| \cdot\left\|\psi_{h}\right\|, \quad \forall w \in H^{1}(\Omega), \forall \psi_{h} \in U_{h}
\end{gathered}
$$

\section{Existence and Uniqueness Analysis for Semi-Discrete Scheme}

In this part, we mainly discuss the existence and uniqueness of semi-discrete mixed finite volume element scheme solutions of the original problem (3).

Theorem 4.1. There exist a unique solution $\left(v_{h}, w_{h}\right) \in U_{0 h} \times U_{h}$ to (8) meets:

$$
\max _{0 \leq t \leq T}\left\|v_{h}\right\|+\max _{0 \leq t \leq T}\left\|w_{h}\right\| \leq C\left(\int_{0}^{t}\|f\| \mathrm{d} \tau+\left\|v_{h}(0)\right\|+\left\|w_{h}(0)\right\|\right)
$$

Proof Choosing $\phi_{h}=v_{h}$ in (8) (a), and $\phi_{h}=w_{h}$ in (8) (b), we can obtain:

$$
\left(v_{h t}, \Pi_{h}^{*} v_{h}\right)+\delta B\left(v_{h}, \Pi_{h}^{*} v_{h}\right)+\left(w_{h t}, \Pi_{h}^{*} w_{h}\right)=\left(f, \Pi_{h}^{*} v_{h}\right)
$$

Applying Lemma 3.2 and Lemma 3.3, there exists a constant $C_{1}(>0)$ associated with $\delta$ such that:

$$
\left(v_{h t}, \Pi_{h}^{*} v_{h}\right)+C_{1}\left\|v_{h}\right\|_{1}+\left(w_{h t}, \Pi_{h}^{*} w_{h}\right) \leq \frac{1}{4 C_{1}}\|f\|^{2}+C_{1}\left\|v_{h}\right\|_{1}^{2}
$$

Integrating both sides from 0 to $t$.

$$
\frac{1}{2}\left(v_{h}, \Pi_{h}^{*} v_{h}\right)+\frac{1}{2}\left(w_{h}, \Pi_{h}^{*} w_{h}\right) \leq C\|f\|^{2}+\left\|v_{h}(0)\right\|^{2}+\left\|w_{h}(0)\right\|^{2}
$$

Applying Lemma 3.2 and Lemma 3.3:

$$
\frac{1}{16}\left\|v_{h}\right\|^{2}+\frac{1}{16}\left\|v_{h}\right\|^{2} \leq C\left(\int_{0}^{t}\|f\|^{2} \mathrm{~d} \tau+\left\|v_{h}(0)\right\|^{2}+\left\|w_{h}(0)\right\|^{2}\right)
$$

Taking the square root of both sides to get the conclusion:

$$
\max _{0 \leq t \leq T}\left\|v_{h}\right\|+\max _{0 \leq t \leq T}\left\|w_{h}\right\| \leq C\left(\int_{0}^{t}\|f\| \mathrm{d} \tau+\left\|v_{h}(0)\right\|+\left\|w_{h}(0)\right\|\right)
$$

To prove the existence and uniqueness of the solution of the system (8), we need only consider its corresponding homogeneous problem:

$$
\left\{\begin{array}{l}
\left(v_{h}, \Pi_{h}^{*} \phi_{h}\right)+\delta B\left(v_{h}, \Pi_{h}^{*} \phi_{h}\right)-B\left(w_{h}, \Pi_{h}^{*} \phi_{h}\right)=0, \quad \forall \phi_{h} \in U_{0 h} \\
\left(w_{h t}, \Pi_{h}^{*} \phi_{h}\right)+B\left(v_{h}, \Pi_{h}^{*} \phi_{h}\right)=0, \quad \forall \phi_{h} \in U_{h} \\
v_{h}(x, 0)=0, \quad \forall x \in \Omega \\
w_{h}(x, 0)=0, \quad \forall x \in \Omega
\end{array}\right.
$$


According to (9), we can easily deduce that $v_{h}=w_{h}=0$, which achieves the uniqueness proof. Since the differential equation is finite dimensional, the uniqueness holds that the existence holds.

\section{Convergence Analysis for Semi-Discrete Scheme}

In order to analyze the error of the scheme, we introduce the mixed finite volume element elliptic projection of the original problem: find $\left(\tilde{v}_{h}, \tilde{w}_{h}\right) \in U_{0 h} \times U_{h}$, meets:

$$
\begin{cases}B\left(\tilde{w}_{h}-w, \Pi_{h}^{*} \phi_{h}\right)=0, & \forall \phi_{h} \in U_{0 h} \\ B\left(\tilde{v}_{h}-v, \Pi_{h}^{*} \phi_{h}\right)=-\left(\tilde{w}_{h}-w, \Pi_{h}^{*} \phi_{h}\right), & \forall \phi_{h} \in U_{h}\end{cases}
$$

Under the condition that $\mathfrak{J}_{h}$ is C-uniform partition, the elliptic projection is unique, and meets the following error estimation formulas [27]:

$$
\begin{gathered}
\left|v-\tilde{v}_{h}\right|_{1}+\left\|w-\tilde{w}_{h}\right\| \leq C h\left(\|v\|_{3}+\|w\|_{2}\right) \\
\left\|v-\tilde{v}_{h}\right\|_{0} \leq C h\left(\|v\|_{3}+\|w\|_{2}\right)
\end{gathered}
$$

We split the errors:

$$
\begin{aligned}
& v_{h}-v=\left(v_{h}-\tilde{v}_{h}\right)+\left(\tilde{v}_{h}-v\right)=\xi+\eta \\
& w_{h}-w=\left(w_{h}-\tilde{w}_{h}\right)+\left(\tilde{w}_{h}-w\right)=\rho+\theta
\end{aligned}
$$

There are:

$$
\begin{aligned}
& \left\|\eta_{t}\right\|=\left\|\tilde{v}_{h t}-v_{t}\right\| \leq C h\left(\left\|v_{t}\right\|_{3}+\left\|w_{t}\right\|_{2}\right) \\
& \left\|\theta_{t}\right\|=\left\|\tilde{w}_{h t}-w_{t}\right\| \leq C h\left(\left\|v_{t}\right\|_{3}+\left\|w_{t}\right\|_{2}\right)
\end{aligned}
$$

From (7) (8) and combining with the elliptic projection (11), we get the following error equations:

$\begin{cases}\text { (a) } B\left(\xi, \Pi_{h}^{*} \phi_{h}\right)-\left(\theta, \Pi_{h}^{*} \phi_{h}\right)+\left(\rho_{t}, \Pi_{h}^{*} \phi_{h}\right)+\left(\theta_{t}, \Pi_{h}^{*} \phi_{h}\right)=0, & \forall \phi_{h} \in U_{0 h} \\ \text { (b) }\left(\xi_{t}, \Pi_{h}^{*} \phi_{h}\right)+\left(\eta_{t}, \Pi_{h}^{*} \phi_{h}\right)+\delta B\left(\xi, \Pi_{h}^{*} \phi_{h}\right)-\delta\left(\theta, \Pi_{h}^{*} \phi_{h}\right)-B\left(\rho, \Pi_{h}^{*} \phi_{h}\right)=0, & \forall \phi_{h} \in U_{h}\end{cases}$

Theorem 5.1. Under the condition that $\mathfrak{J}_{h}$ is C-uniform division, let $(v, w)$ and $\left(v_{h}, w_{h}\right)$ be solutions of (7) and (8), respectively. $v, w$ meets the required regularity condition, then there exist a constant $C>0$ independent of the partition $\mathfrak{I}_{h}$, such that:

$$
\left|v_{h}-v\right|_{1}+\left\|w_{h}-w\right\| \leq C h\left\{\|v\|_{3}+\|w\|_{2}+\left[\int_{0}^{t}\left(\|v\|_{3}^{2}+\|w\|_{2}^{2}+\left\|v_{t}\right\|_{3}^{2}+\left\|w_{t}\right\|_{2}^{2}\right) \mathrm{d} \tau\right]^{\frac{1}{2}}\right\}
$$

Proof Choosing $\phi_{h}=\rho$ in the error Equation (12) (a), and $\phi_{h}=\xi$ in (12) (b) to obtain:

$$
\begin{aligned}
& \left(\xi_{t}, \Pi_{h}^{*} \xi\right)+\left(\eta_{t}, \Pi_{h}^{*} \xi\right)+\delta B\left(\xi, \Pi_{h}^{*} \xi\right)-\delta\left(\theta, \Pi_{h}^{*} \xi\right) \\
& -\left(\theta, \Pi_{h}^{*} \rho\right)+\left(\rho_{t}, \Pi_{h}^{*} \rho\right)+\left(\theta_{t}, \Pi_{h}^{*} \rho\right)=0
\end{aligned}
$$


Applying Lemma 3.3 and Young's inequality, there exists a constant $C_{2}(>0)$ associated with $\delta$ such that:

$$
\begin{aligned}
& C_{2}\|\xi\|_{1}^{2}+\frac{1}{2} \frac{\mathrm{d}}{\mathrm{d} t}\left(\xi, \Pi_{h}^{*} \xi\right)+\frac{1}{2} \frac{\mathrm{d}}{\mathrm{d} t}\left(\rho, \Pi_{h}^{*} \rho\right) \\
& \leq C\left\|\eta_{t}\right\|^{2}+\frac{1}{4 C}\|\xi\|^{2}+\frac{1}{4 C_{2}}\|\theta\|^{2}+C_{2}\|\xi\|_{1}^{2}+C\|\theta\|^{2}+\frac{1}{4 C}\|\rho\|^{2}+C\left\|\theta_{t}\right\|^{2}+\frac{1}{4 C}\|\rho\|^{2}
\end{aligned}
$$

Integrating both sides from 0 to $t$, we have:

$$
\begin{aligned}
& \frac{1}{16}\|\xi\|^{2}+\frac{1}{16}\|\rho\|^{2} \\
& \leq C\left[\int_{0}^{t}\left(\left\|\eta_{t}\right\|^{2}+\|\theta\|^{2}+\left\|\theta_{t}\right\|^{2}\right) \mathrm{d} \tau\right]+C \int_{0}^{t}\|\xi\|^{2} \mathrm{~d} \tau+C \int_{0}^{t}\|\rho\|^{2} \mathrm{~d} \tau
\end{aligned}
$$

By Gronwall's lemma and error estimation of elliptic projection, we obtain:

$$
\|\xi\|^{2}+\|\rho\|^{2} \leq C h^{2}\left[\int_{0}^{t}\left(\left\|v_{t}\right\|_{3}^{2}+\left\|w_{t}\right\|_{2}^{2}+\|v\|_{3}^{2}+\|w\|_{2}^{2}\right) \mathrm{d} \tau\right]
$$

From (12) we can prove that:

$$
\|\rho\| \leq C h\left\{\left[\int_{0}^{t}\left(\left\|v_{t}\right\|_{3}^{2}+\left\|w_{t}\right\|_{2}^{2}+\|v\|_{3}^{2}+\|w\|_{2}^{2}\right) \mathrm{d} \tau\right]^{\frac{1}{2}}\right\}
$$

Then we estimate $|\xi|_{1}$.

Choosing $\phi_{h}=\xi$ in the error Equation (12) (a):

$$
B\left(\xi, \Pi_{h}^{*} \xi\right)-\left(\theta, \Pi_{h}^{*} \xi\right)+\left(\rho_{t}, \Pi_{h}^{*} \xi\right)+\left(\theta_{t}, \Pi_{h}^{*} \xi\right)=0
$$

Applying Lemma 3.3 and Young's inequality:

$$
\frac{1}{2}|\xi|_{1}^{2} \leq C\|\theta\|^{2}+\frac{1}{3}\|\xi\|^{2}+C\left\|\rho_{t}\right\|^{2}+\frac{1}{3}\|\xi\|^{2}+C\left\|\theta_{t}\right\|^{2}+\frac{1}{3}\|\xi\|^{2}
$$

It can be easily known from the error estimation of (16) and elliptic projection that:

$$
|\xi|_{1} \leq C h\left\{\|v\|_{3}+\|w\|_{2}+\left[\int_{0}^{t}\left(\|v\|_{3}^{2}+\|w\|_{2}^{2}+\left\|v_{t}\right\|_{3}^{2}+\left\|w_{t}\right\|_{2}^{2}\right) \mathrm{d} \tau\right]^{\frac{1}{2}}\right\}
$$

Combining (15), (16) and applying triangle inequality, the conclusion can be obtained.

\section{Fully-Discrete Mixed Finite Volume Element Scheme and Convergence Analysis}

Firstly, the spatial region $\Omega$ is divided into $\mathfrak{I}_{h}$ and $\mathfrak{I}_{h}^{*}$ as in Section 2, let $0<t_{0}<t_{1}<\cdots<t_{M}=T$ be a given partition of time interval, then we have the time step $\Delta t=\frac{T}{M}$, and $t^{n}=n \Delta t, n=0,1, \cdots, M$. For a smooth function $v_{h}$, we denote $v_{h}^{n}=v_{h}\left(t^{n}\right)$.

By using the backward Euler scheme of the time derivative, we construct the 
following fully-discrete scheme: Find $\left(v_{h}^{n}, w_{h}^{n}\right) \in U_{0 h} \times U_{h},(n=1,2, \cdots)$, such that:
(a) $\left(\frac{v_{h}^{n}-v_{h}^{n-1}}{\Delta t}, \Pi_{h}^{*} \phi_{h}\right)+\delta B\left(v_{h}^{n}, \Pi_{h}^{*} \phi_{h}\right)-B\left(w_{h}^{n}, \Pi_{h}^{*} \phi_{h}\right)=\left(f^{n}, \Pi_{h}^{*} \phi_{h}\right), \quad \forall \phi_{h} \in U_{0 h}$
(b) $\left(\frac{w_{h}^{n}-w_{h}^{n-1}}{\Delta t}, \Pi_{h}^{*} \phi_{h}\right)+B\left(v_{h}^{n}, \Pi_{h}^{*} \phi_{h}\right)=0$,
$\forall \phi_{h} \in U_{h}$
(c) $v_{h}^{0}(x, 0)=\psi_{h}(x), \quad \forall x \in \Omega$
(d) $w_{h}^{0}(x, 0)=\varphi_{h}^{\prime \prime}(x), \quad \forall x \in \Omega$

Since the problem is a system of linear equations, it is easy to prove that the solution of the problem is unique.

Then we introduce the elliptic projection of the mixed volume element of the original problem, find $\left(\tilde{v}_{h}^{n}, \tilde{w}_{h}^{n}\right):[0, T] \rightarrow U_{0 h} \times U_{h}$, meets:

$$
\begin{cases}B\left(\tilde{w}_{h}^{n}-w^{n}, \Pi_{h}^{*} \phi_{h}\right)=0, & \forall \phi_{h} \in U_{0 h} \\ B\left(\tilde{v}_{h}^{n}-v^{n}, \Pi_{h}^{*} \phi_{h}\right)=-\left(\tilde{w}_{h}^{n}-w^{n}, \Pi_{h}^{*} \phi_{h}\right), & \forall \phi_{h} \in U_{h}\end{cases}
$$

Under the condition that $\mathfrak{I}_{h}$ is C-uniform partition, the elliptic projection is unique, and meets the following error estimation formulas [27]:

$$
\begin{gathered}
\left|v^{n}-\tilde{v}_{h}^{n}\right|_{1}+\left\|w^{n}-\tilde{w}_{h}^{n}\right\| \leq C h\left(\|v\|_{3}+\|w\|_{2}\right) \\
\left\|v^{n}-\tilde{v}_{h}^{n}\right\| \leq C h\left(\|v\|_{3}+\|w\|_{2}\right)
\end{gathered}
$$

Similar to the notations in Section 5, we still denote that:

$$
\begin{aligned}
v_{h}^{n}-v^{n} & =\left(v_{h}^{n}-\tilde{v}_{h}^{n}\right)+\left(\tilde{v}_{h}^{n}-v^{n}\right)=\xi^{n}+\eta^{n} \\
w_{h}^{n}-w^{n} & =\left(w_{h}^{n}-\tilde{w}_{h}^{n}\right)+\left(\tilde{w}_{h}^{n}-w^{n}\right)=\rho^{n}+\theta^{n} \\
\partial_{t} \xi^{n} & =\frac{\xi^{n}-\xi^{n-1}}{\Delta t}, \quad \partial_{t} \eta^{n}=\frac{\eta^{n}-\eta^{n-1}}{\Delta t} \\
\partial_{t} \rho^{n} & =\frac{\rho^{n}-\rho^{n-1}}{\Delta t}, \quad \partial_{t} \theta^{n}=\frac{\theta^{n}-\theta^{n-1}}{\Delta t}
\end{aligned}
$$

Then, we have:

$$
\begin{aligned}
& \left\|\eta_{t}^{n}\right\|=\left\|\tilde{v}_{h t}^{n}-v_{t}^{n}\right\| \leq C h\left(\left\|v_{t}\right\|_{3}+\left\|w_{t}\right\|_{2}\right) \\
& \left\|\theta_{t}^{n}\right\|=\left\|\tilde{w}_{h t}^{n}-w_{t}^{n}\right\| \leq C h\left(\left\|v_{t}\right\|_{3}+\left\|w_{t}\right\|_{2}\right)
\end{aligned}
$$

Based on (7), (8) and elliptic projection (18), the following error equation can be obtained:

$$
\left\{\begin{aligned}
\text { (a) } & B\left(\xi^{n}, \Pi_{h}^{*} \phi_{h}\right)-\left(\theta^{n}, \Pi_{h}^{*} \phi_{h}\right)+\left(\partial_{t} \rho^{n}, \Pi_{h}^{*} \phi_{h}\right)+\left(\partial_{t} \theta^{n}, \Pi_{h}^{*} \phi_{h}\right) \\
= & \left(w_{t}^{n}-\partial_{t} w^{n}, \Pi_{h}^{*} \phi_{h}\right), \quad \forall \phi_{h} \in U_{0 h} \\
\text { (b) } & \delta B\left(\xi^{n}, \Pi_{h}^{*} \phi_{h}\right)-\delta\left(\theta^{n}, \Pi_{h}^{*} \phi_{h}\right)-B\left(\rho^{n}, \Pi_{h}^{*} \phi_{h}\right)+\left(\partial_{t} \xi^{n}, \Pi_{h}^{*} \phi_{h}\right)+\left(\partial_{t} \eta^{n}, \Pi_{h}^{*} \phi_{h}\right) \\
= & \left(v_{t}^{n}-\partial_{t} v^{n}, \Pi_{h}^{*} \phi_{h}\right), \quad \forall \phi_{h} \in U_{h}
\end{aligned}\right.
$$


Theorem 6.1. Under the condition that $\mathfrak{I}_{h}$ is C-uniform division, If $(v, w)$ is a solution to problem (4) or its equivalent form (6) and $v, w$ meet the required regularity condition. Then the solution $\left(v_{h}, w_{h}\right) \in U_{0 h} \times U_{h}$ of the fully discrete mixed finite volume element scheme (17) converges to $(v, w)$, and there exists a positive constant $C$ which does not depend on the subdivision of $\mathfrak{I}_{h}$ meeting the following estimation:

$$
\begin{aligned}
& \max _{0 \leq n \leq T / N}\left|v_{h}^{n}-v^{n}\right|_{1} \leq C(h+\Delta t) \\
& \max _{0 \leq n \leq T / N}\left\|w_{h}^{n}-w^{n}\right\| \leq C(h+\Delta t)
\end{aligned}
$$

Proof Choose $\phi_{h}=\rho^{n}$ in (21) (a) and $\phi_{h}=\xi^{n}$ in (21) (b) to obtain:

$$
\begin{aligned}
& \delta B\left(\xi^{n}, \Pi_{h}^{*} \xi^{n}\right)-\delta\left(\theta^{n}, \Pi_{h}^{*} \xi^{n}\right)+\left(\partial_{t} \xi^{n}, \Pi_{h}^{*} \xi^{n}\right)+\left(\partial_{t} \eta^{n}, \Pi_{h}^{*} \xi^{n}\right) \\
& +\left(\partial_{t} \rho^{n}, \Pi_{h}^{*} \rho^{n}\right)+\left(\partial_{t} \theta^{n}, \Pi_{h}^{*} \rho^{n}\right) \\
& =\left(\theta^{n}, \Pi_{h}^{*} \rho^{n}\right)+\left(v_{t}^{n}-\partial_{t} v^{n}, \Pi_{h}^{*} \xi^{n}\right)+\left(w_{t}^{n}-\partial_{t} w^{n}, \Pi_{h}^{*} \rho^{n}\right)
\end{aligned}
$$

Transposition:

$$
\begin{aligned}
& \frac{\delta}{2}|\xi|_{1}^{2}+\left(\partial_{t} \xi^{n}, \Pi_{h}^{*} \xi^{n}\right)+\left(\partial_{t} \rho^{n}, \Pi_{h}^{*} \rho^{n}\right) \\
& =\left(\theta^{n}, \Pi_{h}^{*} \rho^{n}\right)+\delta\left(\theta^{n}, \Pi_{h}^{*} \xi^{n}\right)+\left|\left(\partial_{t} \eta^{n}, \Pi_{h}^{*} \xi^{n}\right)\right|+\left|\left(\partial_{t} \theta^{n}, \Pi_{h}^{*} \rho^{n}\right)\right| \\
& \quad+\left(v_{t}^{n}-\partial_{t} v^{n}, \Pi_{h}^{*} \xi^{n}\right)+\left(w_{t}^{n}-\partial_{t} w^{n}, \Pi_{h}^{*} \rho^{n}\right)
\end{aligned}
$$

Applying Lemma 3.3:

$$
\begin{aligned}
\left(\partial_{t} \xi^{n}, \Pi_{h}^{*} \xi^{n}\right) & =\frac{1}{\Delta t}\left(\xi^{n}-\xi^{n-1}, \Pi_{h}^{*} \xi^{n}\right) \\
& \geq \frac{1}{2 \Delta t}\left[\left(\xi^{n}, \Pi_{h}^{*} \xi^{n}\right)-\left(\xi^{n-1}, \Pi_{h}^{*} \xi^{n-1}\right)+\left\|\xi^{n}-\xi^{n-1}\right\|^{2}\right] \\
& \geq \frac{1}{2 \Delta t}\left[\left(\xi^{n}, \Pi_{h}^{*} \xi^{n}\right)-\left(\xi^{n-1}, \Pi_{h}^{*} \xi^{n-1}\right)\right]
\end{aligned}
$$

Similarly:

$$
\left(\partial_{t} \rho^{n}, \Pi_{h}^{*} \rho^{n}\right) \geq \frac{1}{2 \Delta t}\left[\left(\rho^{n}, \Pi_{h}^{*} \rho^{n}\right)-\left(\rho^{n-1}, \Pi_{h}^{*} \rho^{n-1}\right)\right]
$$

Combined with Lemma 3.3, there is:

$$
\begin{aligned}
& \frac{1}{2 \Delta t}\left[\left(\xi^{n}, \Pi_{h}^{*} \xi^{n}\right)-\left(\xi^{n-1}, \Pi_{h}^{*} \xi^{n-1}\right)\right] \\
& +\frac{1}{2 \Delta t}\left[\left(\rho^{n}, \Pi_{h}^{*} \rho^{n}\right)-\left(\rho^{n-1}, \Pi_{h}^{*} \rho^{n-1}\right)\right]+C_{3}\left\|\xi^{n}\right\|_{1}^{2} \\
& \leq\left(\theta^{n}, \Pi_{h}^{*} \rho^{n}\right)+\delta\left(\theta^{n}, \Pi_{h}^{*} \xi^{n}\right)+\left|\left(\partial_{t} \eta^{n}, \Pi_{h}^{*} \xi^{n}\right)\right|+\left|\left(\partial_{t} \theta^{n}, \Pi_{h}^{*} \rho^{n}\right)\right| \\
& \quad+\left(v_{t}^{n}-\partial_{t} v^{n}, \Pi_{h}^{*} \xi^{n}\right)+\left(w_{t}^{n}-\partial_{t} w^{n}, \Pi_{h}^{*} \rho^{n}\right) \\
& =\sum_{i=1}^{6} I_{i}
\end{aligned}
$$

For the estimates of the right-sides of (24), we have:

$$
I_{1}=\left(\theta^{n}, \Pi_{h}^{*} \rho^{n}\right) \leq C\left\|\theta^{n}\right\|^{2}+\frac{1}{4 C}\left\|\rho^{n}\right\|^{2}
$$


Similarly, there exist a constant $C_{3}(>0)$ associated with $\delta$ such that:

$$
\begin{gathered}
I_{2}=\delta\left(\theta^{n}, \Pi_{h}^{*} \xi^{n}\right) \leq \frac{1}{4 C_{3}}\left\|\theta^{n}\right\|^{2}+C_{3}\left\|\xi^{n}\right\|^{2} \leq \frac{1}{4 C_{3}}\left\|\theta^{n}\right\|^{2}+C_{3}\left\|\xi^{n}\right\|_{1}^{2} \\
I_{3}=\left|\left(\partial_{t} \eta^{n}, \Pi_{h}^{*} \xi^{n}\right)\right| \leq C\left\|\partial_{t} \eta^{n}\right\|^{2}+\frac{1}{4 C}\left\|\xi^{n}\right\|^{2}
\end{gathered}
$$

where:

$$
\left\|\partial_{t} \eta^{n}\right\|^{2}=\left\|\frac{1}{\Delta t} \int_{t_{n-1}}^{t_{n}} \eta_{t}(\tau) \mathrm{d} \tau\right\|^{2} \leq \frac{1}{\Delta t} \int_{t_{n-1}}^{t_{n}}\left\|\eta_{t}(\tau)\right\|^{2} \mathrm{~d} \tau
$$

Thus:

$$
I_{3} \leq \frac{C}{\Delta t} \int_{t_{n-1}}^{t_{n}}\left\|\eta_{t}(\tau)^{2}\right\| \mathrm{d} \tau+\frac{1}{4 C}\left\|\xi^{n}\right\|^{2}
$$

Similarly:

$$
\begin{gathered}
I_{4} \leq \frac{C}{\Delta t} \int_{t_{n-1}}^{t_{n}}\left\|\theta_{t}(\tau)^{2}\right\| \mathrm{d} \tau+\frac{1}{4 C}\left\|\rho^{n}\right\|^{2} \\
I_{5}=\left(v_{t}^{n}-\partial_{t} v^{n}, \Pi_{h}^{*} \xi^{n}\right) \leq C\left\|v_{t}^{n}-\partial_{t} v^{n}\right\|^{2}+\frac{1}{4 C}\left\|\xi^{n}\right\|^{2}
\end{gathered}
$$

where:

$$
\begin{aligned}
\left\|v_{t}^{n}-\partial_{t} v^{n}\right\|^{2} & =\left\|v_{t}^{n}-\frac{v^{n}-v^{n-1}}{\Delta t}\right\|^{2}=\left\|\frac{1}{\Delta t} \int_{t_{n-1}}^{t_{n}}\left(\tau-t_{n-1}\right) v_{t t}(\tau) \mathrm{d} \tau\right\|^{2} \\
& \leq\left\|\int_{t_{n-1}}^{t_{n}} v_{t t}(\tau) \mathrm{d} \tau\right\|^{2} \leq \Delta t \int_{t_{n-1}}^{t_{n}}\left\|v_{t t}(\tau)\right\|^{2} \mathrm{~d} \tau
\end{aligned}
$$

Thus:

$$
I_{5} \leq \frac{1}{4 C}\left\|\xi^{n}\right\|^{2}+C \Delta t \int_{t_{n-1}}^{t_{n}}\left\|v_{t t}(\tau)\right\|^{2} \mathrm{~d} \tau
$$

Similarly:

$$
I_{6} \leq \frac{1}{4 C}\left\|\rho^{n}\right\|^{2}+C \Delta t \int_{t_{n-1}}^{t_{n}}\left\|w_{t t}(\tau)\right\|^{2} \mathrm{~d} \tau
$$

Substitute all the above estimates into Equation (24), we obtain:

$$
\begin{aligned}
& \frac{1}{2 \Delta t}\left[\left(\xi^{n}, \Pi_{h}^{*} \xi^{n}\right)-\left(\xi^{n-1}, \Pi_{h}^{*} \xi^{n-1}\right)\right]+\frac{1}{2 \Delta t}\left[\left(\rho^{n}, \Pi_{h}^{*} \rho^{n}\right)-\left(\rho^{n-1}, \Pi_{h}^{*} \rho^{n-1}\right)\right] \\
& \leq C\left\|\theta^{n}\right\|^{2}+\frac{1}{4 C}\left\|\rho^{n}\right\|^{2}+\frac{1}{4 C_{3}}\left\|\theta^{n}\right\|^{2}+\frac{C}{\Delta t} \int_{t_{n-1}}^{t_{n}}\left\|\eta_{t}(\tau)^{2}\right\| \mathrm{d} \tau+\frac{1}{4 C}\left\|\xi^{n}\right\|^{2} \\
& \quad+\frac{C}{\Delta t} \int_{t_{n-1}}^{t_{n}}\left\|\theta_{t}(\tau)^{2}\right\| \mathrm{d} \tau+\frac{1}{4 C}\left\|\rho^{n}\right\|^{2}+\frac{1}{4 C}\left\|\xi^{n}\right\|^{2}+C \Delta t \int_{t_{n-1}}^{t_{n}}\left\|v_{t t}(\tau)\right\|^{2} \mathrm{~d} \tau \\
& \quad+\frac{1}{4 C}\left\|\rho^{n}\right\|^{2}+C \Delta t \int_{t_{n-1}}^{t_{n}}\left\|w_{t t}(\tau)\right\|^{2} \mathrm{~d} \tau
\end{aligned}
$$

Multiplying both sides by $2 \Delta t$, then summing over $n$ results in:

$$
\begin{aligned}
& \left(\xi^{n}, \Pi_{h}^{*} \xi^{n}\right)+\left(\rho^{n}, \Pi_{h}^{*} \rho^{n}\right) \\
& \leq C \Delta t\left(\sum_{j=1}^{n}\left\|\theta^{j}\right\|^{2}+\frac{1}{\Delta t} \int_{0}^{T}\left\|\eta_{t}(\tau)\right\|^{2} \mathrm{~d} \tau+\sum_{j=1}^{n}\left\|\rho^{j}\right\|^{2}+\frac{1}{\Delta t} \int_{0}^{T}\left\|\theta_{t}(\tau)\right\|^{2} \mathrm{~d} \tau\right. \\
& \left.+\sum_{j=1}^{n}\left\|\xi^{j}\right\|^{2}+\Delta t \int_{0}^{T}\left\|v_{t t}(\tau)\right\|^{2} \mathrm{~d} \tau+\Delta t \int_{0}^{T}\left\|w_{t t}(\tau)\right\|^{2} \mathrm{~d} \tau\right)+\left(\xi^{0}, \Pi_{h}^{*} \xi^{0}\right)+\left(\rho^{0}, \Pi_{h}^{*} \rho^{0}\right)
\end{aligned}
$$


When the $\Delta t$ is sufficiently small, applying the discrete Gronwall's lemma [28], we have:

$$
\begin{aligned}
\left\|\xi^{n}\right\|^{2}+\left\|\rho^{n}\right\|^{2} \leq & C \Delta t\left(\sum_{j=1}^{n}\left\|\theta^{j}\right\|^{2}+\frac{1}{\Delta t} \int_{0}^{T}\left\|\eta_{t}(\tau)\right\|^{2} \mathrm{~d} \tau+\frac{1}{\Delta t} \int_{0}^{T}\left\|\theta_{t}(\tau)\right\|^{2} \mathrm{~d} \tau\right. \\
& \left.+\Delta t \int_{0}^{T}\left\|v_{t t}(\tau)\right\|^{2} \mathrm{~d} \tau+\Delta t \int_{0}^{T}\left\|w_{t t}(\tau)\right\|^{2} \mathrm{~d} \tau\right)+\left\|\xi^{0}\right\|^{2}+\left\|\rho^{0}\right\|^{2}
\end{aligned}
$$

where:

$$
\begin{gathered}
\left\|\xi^{0}\right\|=\left\|w_{h}^{0}-\tilde{w}_{h}^{0}\right\| \leq\left\|w_{h}^{0}-w^{0}\right\|+\left\|w^{0}-\tilde{w}_{h}^{0}\right\| \leq C h \\
\left\|\rho^{0}\right\|=\left\|v_{h}^{0}-\tilde{v}_{h}^{0}\right\| \leq\left\|v_{h}^{0}-v^{0}\right\|+\left\|v^{0}-\tilde{v}_{h}^{0}\right\| \leq C h
\end{gathered}
$$

By the error estimation of elliptic projection, we obtain:

$$
\left\|\xi^{n}\right\|^{2}+\left\|\rho^{n}\right\|^{2} \leq C\left[(\Delta t)^{2}+h^{2}\right]
$$

From (25) we can prove that:

$$
\left\|\rho^{n}\right\| \leq C(\Delta t+h)
$$

Choosing $\phi_{h}=\xi^{n}$ in (21) (a):

$$
\left(\partial_{t} \rho^{n}, \Pi_{h}^{*} \xi^{n}\right)+B\left(\xi^{n}, \Pi_{h}^{*} \xi^{n}\right)+\left(\partial_{t} \theta^{n}, \Pi_{h}^{*} \xi^{n}\right)-\left(\theta^{n}, \Pi_{h}^{*} \xi^{n}\right)=\left(w_{t}^{n}-\partial_{t} w^{n}, \Pi_{h}^{*} \xi^{n}\right)
$$

Applying Lemma 3.2, Lemma 3.3:

$$
\frac{1}{2}\left|\xi^{n}\right|_{1}^{2} \leq C\left\|\partial_{t} \rho^{n}\right\|^{2}+C\left\|\partial_{t} \theta^{n}\right\|^{2}+C\left\|\theta^{n}\right\|^{2}+C\left\|w_{t}^{n}-\partial_{t} w^{n}\right\|^{2}+\frac{1}{C}\left\|\xi^{n}\right\|^{2}
$$

For the estimates of the right-sides of (27), we have:

$$
\begin{gathered}
\left\|\partial_{t} \rho^{n}\right\|^{2}=\left\|\frac{1}{\Delta t} \int_{t_{n-1}}^{t_{n}} \rho_{t}(\tau) \mathrm{d} \tau\right\|^{2} \leq \frac{1}{\Delta t} \int_{t_{n-1}}^{t_{n}}\left\|\rho_{t}(\tau)\right\|^{2} \mathrm{~d} \tau \\
\left\|\partial_{t} \theta^{n}\right\|^{2}=\left\|\frac{1}{\Delta t} \int_{t_{n-1}}^{t_{n}} \theta_{t}(\tau) \mathrm{d} \tau\right\|^{2} \leq \frac{1}{\Delta t} \int_{t_{n-1}}^{t_{n}}\left\|\theta_{t}(\tau)\right\|^{2} \mathrm{~d} \tau \\
\left\|w_{t}^{n}-\partial_{t} w^{n}\right\|^{2}=\left\|w_{t}^{n}-\frac{w^{n}-w^{n-1}}{\Delta t}\right\|^{2}=\left\|\frac{1}{\Delta t} \int_{t_{n-1}}^{t_{n}}\left(\tau-t_{n-1}\right) w_{t t}(\tau) \mathrm{d} \tau\right\|^{2} \\
\leq \Delta t \int_{t_{n-1}}^{t_{n}}\left\|w_{t t}(\tau)\right\|^{2} \mathrm{~d} \tau
\end{gathered}
$$

The above inequality leads to:

$$
\begin{aligned}
\left|\xi^{n}\right|_{1}^{2} \leq & C\left(\frac{1}{\Delta t} \int_{t_{n-1}}^{t_{n}}\left\|\rho_{t}(\tau)\right\|^{2} \mathrm{~d} \tau+\frac{1}{\Delta t} \int_{t_{n-1}}^{t_{n}}\left\|\theta_{t}(\tau)\right\|^{2} \mathrm{~d} \tau\right. \\
& \left.+\Delta t \int_{t_{n-1}}^{t_{n}}\left\|w_{t t}(\tau)\right\|^{2} \mathrm{~d} \tau\right)+\frac{1}{C}\left\|\xi^{n}\right\|^{2}
\end{aligned}
$$

Combining (25) and the error estimation of elliptic projection, we derive that:

$$
\left|\xi^{n}\right|_{1} \leq C(\Delta t+h)
$$

Finally, apply (26) (28) and triangle inequality to complete the proof. 


\section{Numerical Simulation}

In this part, we will give two experiments to test our mixed finite volume element scheme. The numerical results will be presented to illustrate the efficiency and order of accuracy of the algorithm.

Example 1: The vibration equation of beam with structural damping is considered as follows:

$$
\begin{cases}\text { (a) } u_{t t}-u_{x x t}+u_{x x x x}=f(x, t), & (x, t) \in(0,1) \times(0,1] \\ \text { (b) } u(x, 0)=\varphi(x), u_{t}(x, 0)=\psi(x), & x \in(0,1) \\ \text { (c) } u(0, t)=u(1, t)=0, u_{x}(0, t)=u_{x}(1, t)=0, & t \in[0,1]\end{cases}
$$

This is a special case of (3), where $\delta=1, T=1, L=1$. We give a exact solution to the problem $u(x, t)=\sin (\pi x) \cos (\pi t)$, then we have:

$$
\begin{gathered}
v(x, t)=-\pi \sin (\pi x) \sin (\pi t) \\
w(x, t)=-\pi^{2} \sin (\pi x) \cos (\pi t) \\
f(x, t)=\left[\pi^{4} \cos (\pi t)-\pi^{3} \sin (\pi t)-\pi^{2} \cos (\pi t)\right] \sin (\pi x)
\end{gathered}
$$

$v, w$ are solved by mixed finite volume element scheme, $u$ can be obtained from $v$ by backward Euler method, Where the spatial step is $h=\frac{1}{N}$, time step is $\Delta t=\frac{1}{M}$, The errors and spatial convergence order of $u, v, w$ are shown in Tables 1-3 respectively when $M=N^{2}$. As can be seen from Tables $1-3$, in the sense of maximum norm, $L^{2}$ norm and $H_{1}$ norm, the displacement, bending moment and velocity of the beam under vibration are better approximated than the theoretical estimations, which justifies the effectiveness of the mixed finite volume element method.

Using MATLAB software, we can get the function figures of the numerical solution and analytical solution, as indicated in Figures 1-6. It proves that the degree of numerical solutions is approximating the exact solutions in different grid points.

Example 2: In Example 1, we assume that the beam is in a free vibration state, choosing $x=0.5, t \in(0,3]$. Different values of $\delta$ were used to verify the

Table 1. The computational errors and convergence orders of $u$.

\begin{tabular}{ccccccc}
\hline$h$ & $\left\|u-u_{h}\right\|_{\infty}$ & order & $\left\|u-u_{h}\right\|_{0, h}$ & order & $\left\|u-u_{h}\right\|_{1, h}$ & order \\
\hline$\frac{1}{2^{4}}$ & $7.379 \mathrm{e}-03$ & - & $5.218 \mathrm{e}-03$ & - & $3.697 \mathrm{e}-02$ & - \\
$\frac{1}{2^{5}}$ & $1.751 \mathrm{e}-03$ & 2.075 & $1.238 \mathrm{e}-03$ & 2.075 & $1.860 \mathrm{e}-02$ & 0.9912 \\
$\frac{1}{2^{6}}$ & $4.319 \mathrm{e}-04$ & 2.019 & $3.054 \mathrm{e}-04$ & 2.019 & $9.469 \mathrm{e}-03$ & 0.9740 \\
$\frac{1}{2^{7}}$ & $1.076 \mathrm{e}-04$ & 2.005 & $7.609 \mathrm{e}-05$ & 2.005 & $4.794 \mathrm{e}-03$ & 0.9820 \\
$\frac{1}{2^{8}}$ & $2.688 \mathrm{e}-05$ & 2.001 & $1.900 \mathrm{e}-05$ & 2.001 & $2.413 \mathrm{e}-03$ & 0.9898 \\
\hline
\end{tabular}




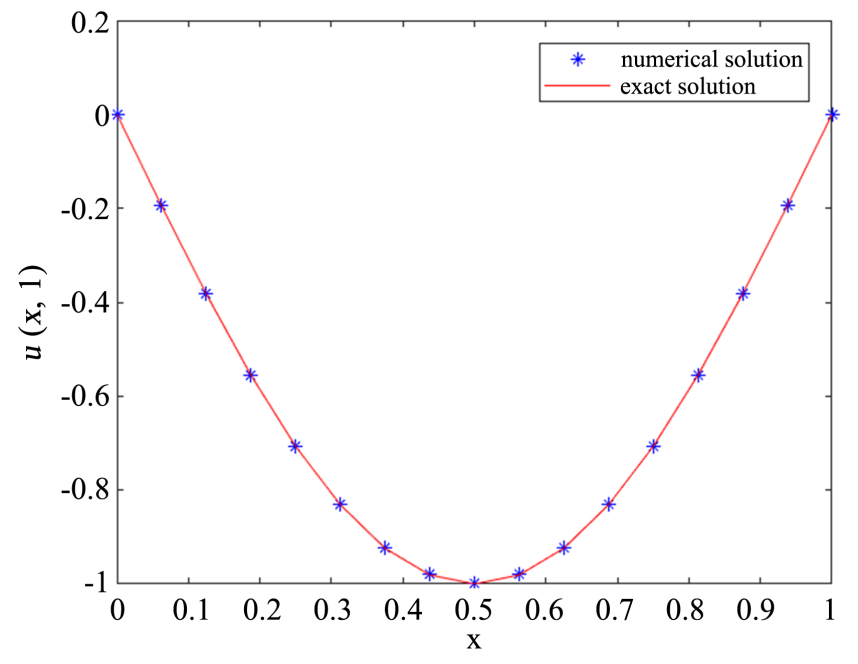

Figure 1. $u$ and $u_{h}$ at $t=1$.

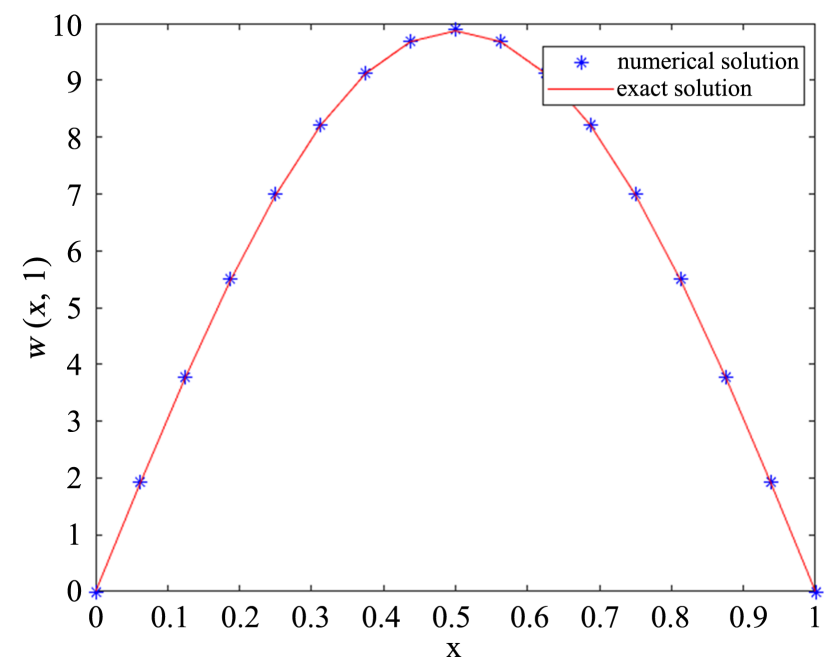

Figure 2. $w$ and $w_{h}$ at $t=1$.

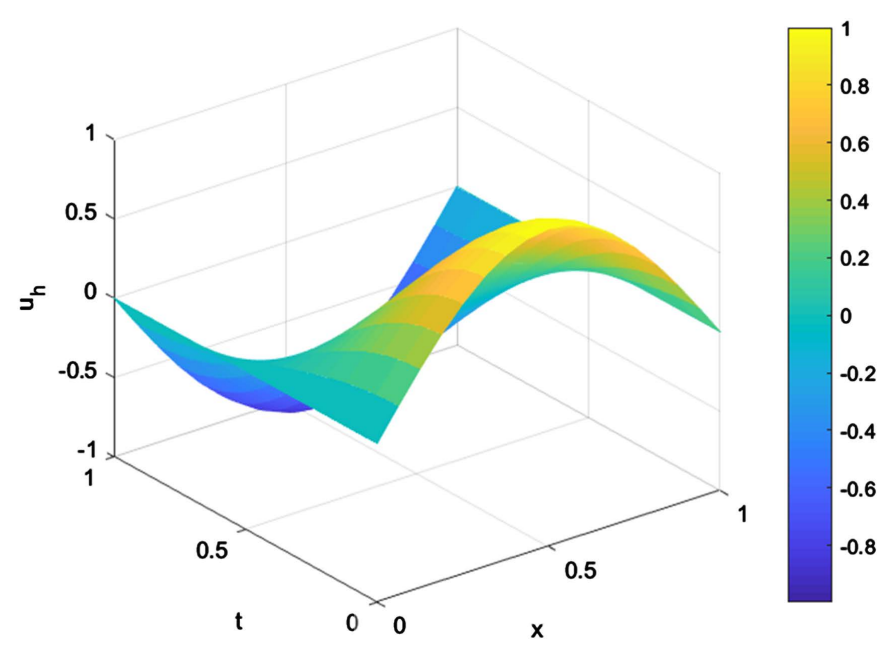

Figure 3. The space-time image of $u_{h}$ when $h=\frac{1}{16}$. 


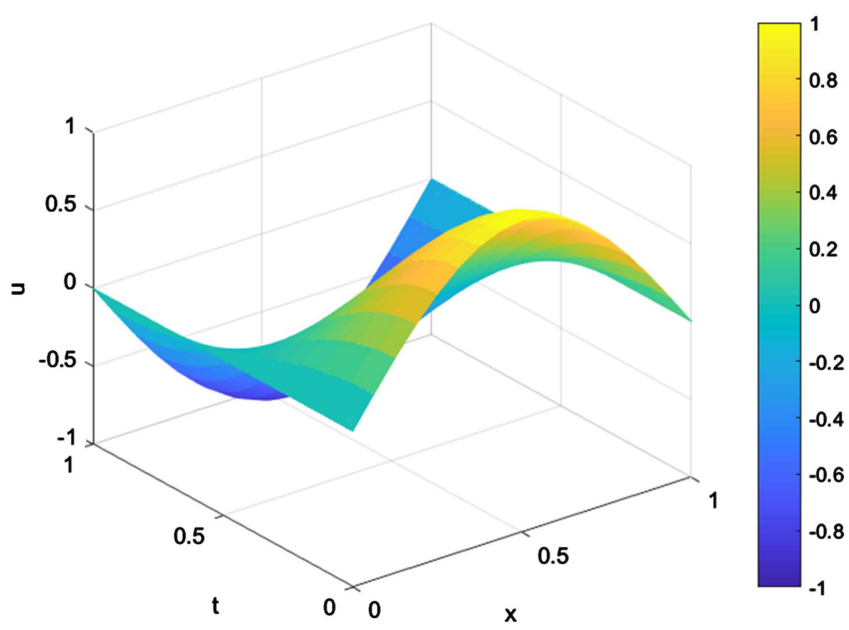

Figure 4. The space-time image of $u$ when $h=\frac{1}{16}$.

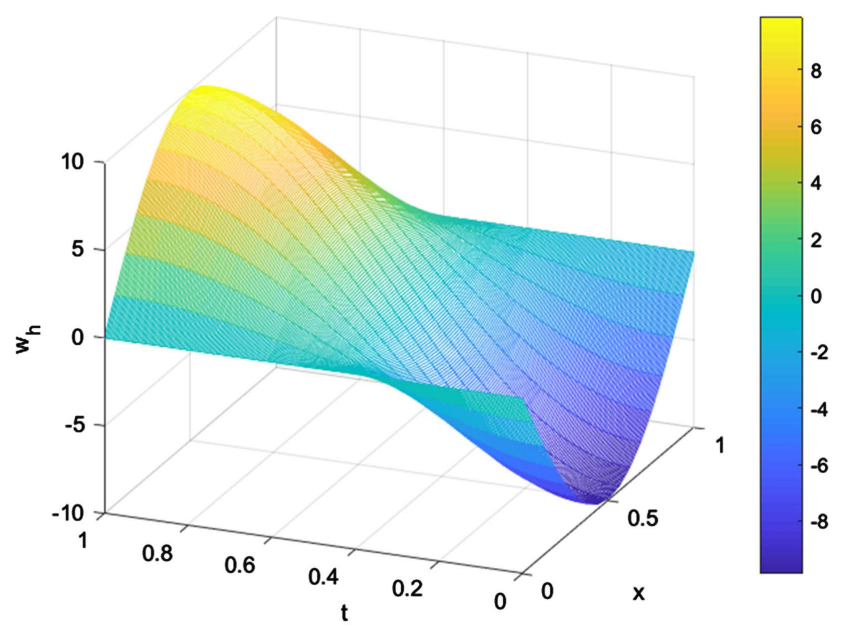

Figure 5. The space-time image of $w_{h}$ when $h=\frac{1}{16}$.

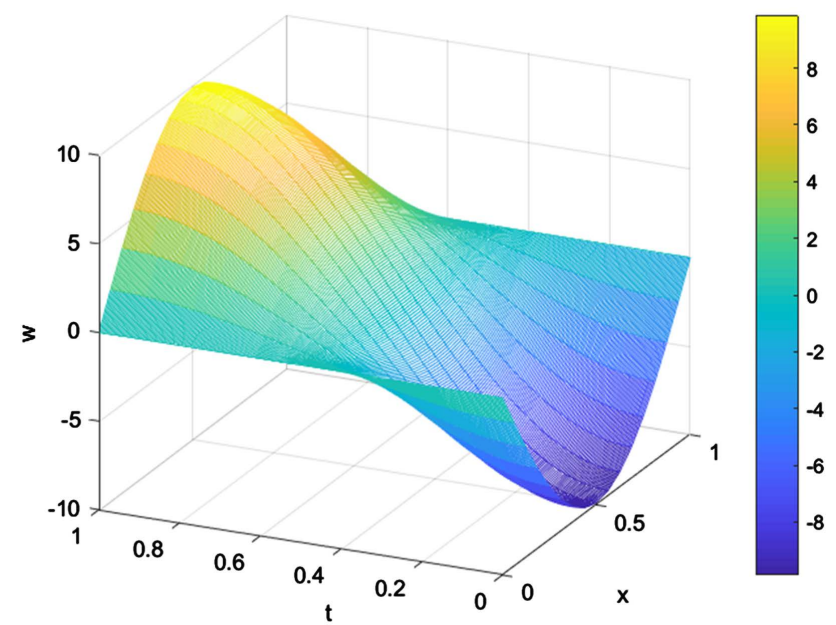

Figure 6. The space-time image of $w$ when $h=\frac{1}{16}$. 
influence of material damping on beam vibration. As can be seen from Figure 7, the larger the damping coefficient of material structure $\delta$ is, the faster the attenuation rate of beam vibration will be.

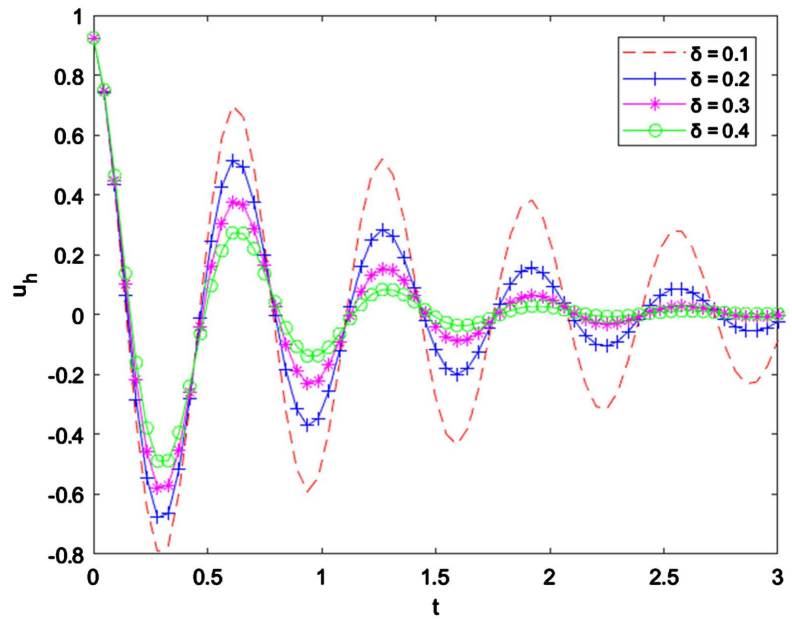

Figure 7. The image of $u(x, t)$ changing with time at midpoint when $\delta$ takes different values.

Table 2. The computational errors and convergence orders of $v$.

\begin{tabular}{ccccccc}
\hline$h$ & $\left\|v-v_{h}\right\|_{\infty}$ & order & $\left\|v-v_{h}\right\|_{0, h}$ & order & $\left\|v-v_{h}\right\|_{1, h}$ & order \\
\hline$\frac{1}{2^{4}}$ & $2.110 \mathrm{e}-02$ & - & $1.492 \mathrm{e}-02$ & - & $2.241 \mathrm{e}-01$ & - \\
$\frac{1}{2^{5}}$ & $5.281 \mathrm{e}-03$ & 1.998 & $3.734 \mathrm{e}-03$ & 1.998 & $1.157 \mathrm{e}-01$ & 0.9531 \\
$\frac{1}{2^{6}}$ & $1.320 \mathrm{e}-03$ & 1.999 & $9.338 \mathrm{e}-04$ & 1.999 & $5.883 \mathrm{e}-02$ & 0.9767 \\
$\frac{1}{2^{7}}$ & $3.302 \mathrm{e}-04$ & 1.999 & $2.334 \mathrm{e}-04$ & 1.999 & $2.965 \mathrm{e}-02$ & 0.9885 \\
$\frac{1}{2^{8}}$ & $8.255 \mathrm{e}-05$ & 2.000 & $5.837 \mathrm{e}-05$ & 2.000 & $1.488 \mathrm{e}-02$ & 0.9943 \\
\hline
\end{tabular}

Table 3. The computational errors and convergence orders of $w$.

\begin{tabular}{ccccccc}
\hline$h$ & $\left\|w-w_{h}\right\|_{\infty}$ & order & $\left\|w-w_{h}\right\|_{0, h}$ & order & $\left\|w-w_{h}\right\|_{1, h}$ & order \\
\hline$\frac{1}{2^{4}}$ & $1.444 \mathrm{e}-02$ & - & $1.021 \mathrm{e}-02$ & - & $1.533 \mathrm{e}-01$ & - \\
$\frac{1}{2^{5}}$ & $3.611 \mathrm{e}-03$ & 1.999 & $2.553 \mathrm{e}-03$ & 1.999 & $7.917 \mathrm{e}-02$ & 0.9541 \\
$\frac{1}{2^{6}}$ & $9.028 \mathrm{e}-04$ & 1.999 & $6.384 \mathrm{e}-04$ & 1.999 & $4.022 \mathrm{e}-02$ & 0.9770 \\
$\frac{1}{2^{7}}$ & $2.257 \mathrm{e}-04$ & 2.000 & $1.596 \mathrm{e}-04$ & 2.000 & $2.027 \mathrm{e}-02$ & 0.9886 \\
$\frac{1}{2^{8}}$ & $5.643 \mathrm{e}-05$ & 2.000 & $3.990 \mathrm{e}-05$ & 2.000 & $1.017 \mathrm{e}-02$ & 0.9943 \\
\hline
\end{tabular}




\section{Conclusions and Suggestions}

In this paper, two intermediate functions with practical significance are introduced to the vibration equation of the structural damping beam. The fourth-order partial differential equation is transformed into a set of second-order partial differential equations. The spatial derivative is discretized by the mixed finite-volume element scheme, and the time derivative is discretized by the backward Euler scheme. A mixed finite-volume element scheme is obtained. The existence and uniqueness of the scheme solution are analyzed and the error estimate is given. Finally, a numerical example is given to verify the accuracy and effectiveness of the scheme. In future work, the derivative of time can be processed with a more precise discrete scheme, and the vibration equations of beams with different damping types can also be solved numerically by this method.

\section{Acknowledgements}

This work is supported in part by the National Natural Science Foundation of China under Grant nos. 11501335 and by a Project of Natural Science Foundation of Shandong Province under Grant nos. ZR2017MA020.

\section{Conflicts of Interest}

The authors declare no conflicts of interest regarding the publication of this paper.

\section{References}

[1] Lazer, A.C. and Mckenna, P.J. (1990) Large-Amplitude Periodic Oscillations in Suspension Bridges: Some New Connections with Nonlinear Analysis. Society for Industrial and Applied Mathematics Review, 32, 537-578.

https://doi.org/10.1137/1032120

[2] Doyle, P.F. and Pavlovic, M.N. (1982) Vibration of Beams on Partial Elastic Foundations. Earthquake Engineering \& Structural Dynamics, 10, 663-674. https://doi.org/10.1002/eqe.4290100504

[3] Cornil, M.B., Capolungo, L., Qu, J.M. and Jairazbhoy, V.A. (2007) Free Vibration of a Beam Subjected to Large Static Deflection. American Journal of Computational Mathematics, 303, 723-740. https://doi.org/10.1016/j.jsv.2007.02.016

[4] Thankane, K.S. and Sty̌̌, T. (2009) Finite Difference Method for Beam Equation with Free Ends Using Mathematica. Southern Africa Journal of Pure and Applied Mathematics, 4, 61-78.

[5] Gupta, C.P. (1990) Existence and Uniqueness Theorems for Some Fourth Order Fully Quasilinear Boundary Value Problems. Applicable Analysis, 36, 157-169. https://doi.org/10.1080/00036819008839930

[6] Gupta, C.P. (1988) Existence and Uniqueness Theorems for the Bending of an Elastic Beam Equation. Applicable Analysis, 26, 289-304. https://doi.org/10.1080/00036818808839715

[7] Gupta, C.P. (1988) Existence and Uniqueness Results for the Bending of an Elastic Beam Equation at Resonance. Advances in Water Resources, 135, 208-225. https://doi.org/10.1016/0022-247X(88)90149-7 
[8] Ni, P. and Gao, Y.X. (1995) A Generalized Difference Method for Beam Vibration Equations (I). Environmental Health Perspectives, 4, 14-19.

[9] Xu, S.J. and Wang, C.H. (2007) A Stable Finite Difference Approximation for Vibration Equation of Beams. Journal of Hydrodynamics, 24, 79-81.

[10] Zeng, W.P. (2002) Four-Level Explicit Difference Schemes with Parameters for Solving the Equation of Fourth-Order rod Vibration. Journal of Huaqiao University (Natural Science), 23, 116-121.

[11] Zeng, W.P. (2003) Two New Classes of Implicit Difference Schemes for Solving Rod Vibration Equation of Fourth-Order. Journal of Huaqiao University (Natural Science), 2, 116-121.

[12] Yin, Z. and Shen, W.F. (2003) A Numerical Simulation Method to Solve the Beam Vibration Problem on Elastic Foundation. Journal of Shandong University of Technology, 5, 582-584.

[13] Seva, A. (2008) Solution of Free Vibration Equations of Beam on Elastic Soil by Using Differential Transform Method. Applied Mathematical Modelling, 32, 1744-1757. https://doi.org/10.1016/j.apm.2007.06.010

[14] Zhou, L.Q., Chen, Y.S. and Chen, F.Q. (2010) Multi-Parameters and High Accuracy Scheme for Beam Vibration Equations. Journal of Anqing Teachers College (Natural Science Edition), 16, 62-65.

[15] Sabitov, K.B. (2017) Cauchy Problem for the Beam Vibration Equation. Differential Equations, 53, 658-664. https://doi.org/10.1134/S0012266117050093

[16] Chen, G. and Russell, D.L. (1980) A Mathematical Model for Linear Elastic Systems with Structural Damping. Quarterly of Applied Mathematics, 39, 32. https://doi.org/10.1090/qam/644099

[17] Fan, H.X. and Li, Y.X. (2014) Analyticity and Exponential Stability of Semigroups for the Elastic Systems with Structural Damping in Banach Spaces. Journal of Mathematical Analysis and Applications, 410, 316-322. https://doi.org/10.1016/j.jmaa.2013.08.028

[18] Fan, H.X., Li, Y.X. and Chen, P.Y. (2013) Existence of Mild Solutions for the Elastic Systems with Structural Damping in Banach Spaces. Abstract and Applied Analysis, 2013, 1-6. https://doi.org/10.1155/2013/746893

[19] Tang, Y. and Yin, Z. (2021) Hermite Finite Element Method for a Class of Viscoelastic Beam Vibration Problem. Engineering, 13, 463-471. https://doi.org/10.4236/eng.2021.138033

[20] Russell, T.F. (1995) Rigorous Block-Centered Discretizations on Irregular Grids: Improved Simulation of Complex Reservior Systems. Technical Report No. 3, Project Report, Resevoir Simulation Research Corporation.

[21] Cai, Z., Jones, J.E., McCormick, S.F. and Russell, T.F. (1997) Control-Volume Mixed Finite Element Methods. Computational Geosciences, 1, 289-315. https://doi.org/10.1023/A:1011577530905

[22] Jones, J.E., Mccormick, S.F., Manteuffel, T.A., Ruge, J.W. and Russell, T.F. (1995) A Mixed Finite Volume Element Method for Accurate Computation of Fluid Velocities in Porous Media. University of Colorado, Denver.

[23] Wang, T.K. (2004) A Mixed Finite Volume Element Method for Solving the Beam Equilibrium Equations. Chinese Journal of Engineering Mathematics, 21, 133-136.

[24] Yuan, Y.R., Yang, Q., Li, C.F. and Sun, T.J. (2013) A Mixed-Finite Volume Element Coupled with the Method of Characteristic Fractional Step Difference for Simulating Transient Behavior of Semiconductor Device of Heat Conductor and Its $\mathrm{Nu}$ - 
merical Analysis. Acta Mathematicae Applicatae Sinica, 4, 221-240.

[25] Yuan, Y.R., Sun, T.J., Li, C.F., Liu, Y.X. and Yang, Q. (2013) Mixed Volume Element Combined with Characteristic Mixed Finite Volume Element Method for Oil-Water Two Phase Displacement Problem. Journal of Computational and Applied Mathematics, 340, 404-419. https://doi.org/10.1016/j.cam.2018.02.038

[26] Li, R.H., Chen, Z.Y. and Wu, W. (2000) The Generalized Difference Methods for Differential Equations. CRC Press, Boca Raton.

https://doi.org/10.1201/9781482270211

[27] Wang, T.K. (2002) The Research on Numerical Algorithms for Several Class of Differential Equations. Shandong University, Jinan.

[28] Li, R.H. and Liu, B. (1997) Numerical Solutions for Differential Equations. 